\title{
Análisis de las preferencias del consumidor por las hortalizas frescas en el Caribe colombiano
}

\section{Analysis of consumer preferences for fresh vegetables in the colombian Caribbean}

\author{
Antonio María Martínez-Reina ${ }^{1 *}$; Lilibet Tordecilla-Zumaqué ${ }^{\oplus}$; \\ Maria del Valle Rodríguez-Pinto ${ }^{1}$; Liliana María Grandett-Martínez $^{1 \odot}$ \\ Recibido para publicación: Marzo 1 de 2019 - Aceptado para publicación: Mayo 24 de 2019
}

\begin{abstract}
RESUMEN
El objetivo de este trabajo fue analizar las preferencias del consumidor al momento de decidir por la compra de hortalizas frescas en la región Caribe, para obtener información organizada que permita tomar decisiones acerca de la posibilidad de ampliar la oferta de estos productos de la agricultura. Se aplicó una encuesta estructurada que incluía variables socioeconómicas del consumidor y otras como frecuencia de compra, principales hortalizas que consumen, sitio de compra, a 308 familias consumidoras localizadas en las siete ciudades principales de la Región Caribe seleccionadas al azar. Para el análisis se utilizaron tablas de contingencia, estadística descriptiva, análisis de correlación y análisis de varianza. Los Resultados permiten apreciar que la zanahoria, la cebolla blanca, la ahuyama y el tomate son las especies hortícolas de mayor preferencia por los consumidores, en promedio 1,79 kilos por compra. El trabajo permite concluir que existe una relación directa entre el aumento del consumo y la edad del consumidor siendo los mayores de 50 años lo que más prefieren las hortalizas, lo cual se explica por el valor nutritivo y los beneficios a la salud. Se evidenció que el sitio de compra está altamente relacionado con el estrato social del consumidor es así como los estratos mas altos prefieren comprar en los supermercados en tanto que los estratos más bajos prefieren comprar en los mercados públicos y en la tienda de barrio.
\end{abstract}

Palabras clave: Consumidor; Ingreso; Satisfacción; Elección; Mercado; Precio.

'Corporación Colombiana de Investigación Agropecuaria Agrosavia. Cereté, Colombia.

*Autor para correspondencia: Antonio Martínez

Email: amartienez@agrosavia.co

\begin{abstract}
The objective of this work was to analyze consumer preferences when deciding on purchasing fresh vegetables in the Caribbean region in order to obtain organized information for decision making about possibility of expanding agricultural product supply. A structured survey with socioeconomic variables for consumer and others such as purchase frequency, main vegetables consumed, purchase site was applied to 308 consumer families randomly selected in the seven main cities of the Caribbean Region. For the analysis, contingency tables, descriptive statistics, correlation analysis and analysis of variance were used. The results allowed to find that carrot, white onion, squash and tomato are the most preferred vegetables with an average 1.79 kilos per purchase. The collected data showed a relation between consumption and consumer age, People 50 year old, and older, prefer more vegetables, bacause of nutritional value and health benefits. It was evidenced that purchase site is highly related to consumer economic level, as higher economic income consumers prefer purchasing in griocery stoores, while low income consumers purchase in public markets and neighborhood markets.
\end{abstract}

Key words: Consumer; Income; Satisfaction; Choice; Market; Price.

\section{Cómo citar}

Martínez-Reina, A., Tordecilla-Zumaqué, L., Rodríguez-Pinto, M. y Grandett-Martínez, L. 2019. Análisis de las preferencias del consumidor por las hortalizas frescas en el Caribe colombiano. Temas Agrarios, 24(2):119-128. https://doi.org/10.21897/rta.v24i2.2070 crear a partir del material, de forma no comercial, dando crédito y licencia de forma adecuada a los autores de la obra. 


\section{INTRODUCCIÓN}

De acuerdo con la Organización de las Naciones Unidas para la Agricultura y la Alimentación FAO (2002) y el Ministerio de Salud y Protección Social (2013) reportan que el $27,9 \%$ de la población colombiana no consume hortalizas y calculan que el consumo por persona día corresponde a 45,75 gramos, que se considera muy bajo comparado con el consumo de hortalizas en Alemania, que es de 255 g/día por persona.

Existen elementos teóricos para estudiar el tema del consumo de bienes en general, los cuales son aplicables al caso de las hortalizas por ejemplo Case et al. (2012) proponen que en la teoría neoclásica del consumo, la demanda está determinada por el precio, nivel de ingreso, el precio de los bienes sustitutos y complementarios, las expectativas, gustos y preferencias. Las variables diferentes a los precios, las cuales son de tipo cualitativo como la edad, genero, costumbres, religión, cultura, generan cambios en las cantidades demandadas de cada consumidor y se definen como los gustos de los consumidores. Ahora bien, las decisiones de consumo dependen de acciones racionales e irracionales, son así mismo el resultado de influencias por factores internos o externos al consumidor buscando aquellos que realmente lo satisfagan (Samuelson, 1948; Parkin, 2006; Salazar, 2010; Reynolds, 2013).

Nicholson (1997), afirma que el consumidor revela sus preferencias de acuerdo con la utilidad, o satisfacción que le proporciona consumir un bien, y está relacionada con la calidad, el consumidor define sus preferencias de acuerdo al nivel de satisfacción y revela estas preferencias. Los individuos son capaces de ordenar todas las situaciones posibles del menos al más deseable y las denominan como utilidad.

Al tratar el tema de las preferencias por el consumo de las hortalizas, no existe una regla única y homogénea que dirija al consumidor a inclinarse por tal bien, sino que obedece a diferentes atributos que él mismo ha definido en forma individual, en concordancia con la teoría neoclásica del consumidor este revela sus preferencias, de acuerdo al grado de satisfacción o utilidad que deriva del consumo del bien en este caso las hortalizas (Miller, 2006).

Martín (2005) en un trabajo sobre preferencias en el consumo de hortalizas frescas en España, afirma que el consumidor decide la compra atendiendo a sus propiedades nutricionales, facilidad culinaria, época del año o estado climático. Igualmente, la estacionalidad de la producción supone variaciones en el consumo de un tipo u otro de hortalizas. La frecuencia en la compra también varía de acuerdo al uso que se da, sea para el consumo en fresco o preparada.

En la Región Caribe, no se cuenta con información documentada en forma explícita y sistemática sobre el consumo de hortalizas frescas, esto dificulta la toma de decisiones tanto de producción como de investigación, el objetivo de este trabajo fue analizar las preferencias y el perfil del consumidor de hortalizas en la Región Caribe de Colombia, para entender las posibilidades de aumentar las áreas cultivadas en la medida que haya expectativas del crecimiento del consumo.

La hipótesis de investigación plantea que las posibilidades del aumento de la producción solo tienen lugar si se conocen las preferencias de los consumidores por las hortalizas frescas en la Región Caribe de Colombia.

\section{MATERIALES Y MÉTODOS}

Utilizando el método inductivo exploratorio, se consideró al consumidor de manera individual para inferir el comportamiento del consumo en la Región Caribe. La muestra definida fueron 308 consumidores elegidos al azar en las ciudades de Montería, Sincelejo, Cartagena, Barranquilla, Santa Marta, Valledupar y Riohacha. Con estos datos se estableció el orden de preferencias y perfil del consumidor, de hortalizas frescas. 
Para la recolección de la información en campo, se diseñó un formulario de encuesta que constó de 18 preguntas y 8 variables en total, que correspondieron a los datos socioeconómicos del consumidor, frecuencia de compra, nivel de ingresos, responsable de las compras, sitios de compra, cantidades compradas, preferencias y posibilidades de aumentar el consumo.

Para determinar el tamaño de la muestra se usó el muestreo aleatorio simple, no fue posible definir el número total de hogares para las principales ciudades de la Región Caribe, por lo que fue necesario aplicar la fórmula de muestreo cuando no se conoce el tamaño de la población de acuerdo con (Torres, 2016) la cual se plantea de la siguiente forma:

$$
\mathrm{n}=\frac{\left(\mathrm{Z}^{2 *} \mathrm{P}^{*} \mathrm{Q}\right)}{\mathrm{d}^{2}}
$$

Donde:

$Z$ = nivel de confianza,

$\mathrm{P}=$ probabilidad de éxito, o proporción esperada

$\mathrm{Q}=$ probabilidad de fracaso

$\mathrm{d}=$ error máximo de muestreo permitido

En este caso:

$$
\mathrm{n}=\frac{1.96^{2 *} 0,05 * 0.95}{(0,0246)^{2}}=306
$$

El cálculo dio un total de 306 encuestas, no obstante, se realizaron para este estudio 308 encuestas distribuidas de acuerdo con la densidad de población, aplicando 65 encuestas en la ciudad de Barranquilla, 47 en Cartagena, 64 en Montería, 43 en Sincelejo, 39 en Santa Marta, 20 en Riohacha y 30 en Valledupar.

Se aplicaron encuestas a los responsables de las compras de hortalizas en las familias de las siete principales ciudades de la Región Caribe, en los supermercados, mercados público y en algunos casos directamente a las casas puerta a puerta y en las oficinas de entidades públicas.
En este estudio se utilizaron tablas de contingencia para establecer las relaciones de las variables que explican el consumo de hortalizas en la Región Caribe de Colombia de acuerdo a Rodríguez y Mora (2001), además una análisis de varianza para realizar las comparaciones de variable cuantitativa, como cantidades demandadas de hortalizas a través de las variables categóricas: genero (hombre o mujer), tamaño de la familia y nivel de ingreso se hizo uso de la técnica de análisis de varianza ANOVA de acuerdo con Hair et al. (1999) Ilegando así a una respuesta de la variable dependiente a través de las variables independientes de tipo categórico o cualitativas que se presenta en la siguiente ecuación:

$$
\mathrm{Y}_{1}+\mathrm{Y}_{2}+\mathrm{Y}_{3}+\mathrm{Yn}=\mathrm{X}_{1}+\mathrm{X}_{2}+\mathrm{X}_{3}+\mathrm{Xn}
$$

Donde

$\mathrm{Yn}=$ Variable dependiente cuantitativa o métrica

$\mathrm{Xn}=$ Variables independientes, categóricas o cualitativas.

La hipótesis nula asume que las medias poblacionales son iguales, mientras que la hipótesis alternativa asume que estas son distintas. En otras palabras:

$$
\mathrm{H}_{0}: \mu_{0}=\mu_{1}=\mu_{2}=\mu_{\mathrm{c}}
$$

La hipótesis nula se puede probar por medio del estadístico $F$, que es una distribución de la probabilidad de las relaciones de la varianza de la muestra y sigue una distribución (c-1) y $(\mathrm{N}-\mathrm{c})$ grados de libertad (df) (Malhotra, 1997).

\section{RESULTADOS Y DISCUSIÓN}

\section{Características socioeconómicas de la muestra}

La muestra estuvo integrada principalmente por mujeres $(73 \%)$, que corresponde a la persona responsable de las compras en el hogar, en tanto que el $27 \%$ de los encuestados son hombres y también tienen la responsabilidad de las compras en la familia. La edad promedio de los encuestados se 
encuentra entre los 40 a 50 años, la moda es de 42 años, lo que se considera un estado de población madura. Con relación a la escolaridad del jefe de familia, se encontró que el 55\% no tienen estudios universitarios en tanto que un $45 \%$ cuenta con estudios superiores. En promedio las familias se componen de cuatro miembros.

Con relación a la ocupación u oficio el $38 \%$ son empleados asalariados, el $30 \%$ independientes, el $27 \%$ amas de casa y un $5 \%$ tienen otra ocupación, en conclusión, predomina más los empleados asalariados. El nivel de ingresos promedio mensual de las familias está en un rango de \$500.000 y $\$ 1.000 .000$ peso colombiano (COP), que corresponde al $43 \%$ de la muestra encuestada.

El $100 \%$ de la muestra incluyen en la compra algún producto clasificado como hortalizas. Sin embargo, las cantidades compradas en promedio son de $1,79 \mathrm{k}$ y las hortalizas que más se repiten para una frecuencia semanal son ahuyama, ají dulce, arveja, berenjena, calabaza, cebolla cabezona blanca, cebolla cabezona roja, cebolla junca, frijol cabecita negra, frijol cargamanto, habichuela corta, frijol zaragoza, lechuga, lenteja, pepino, cebolla puerro, rábano, remolacha, repollo, tomate, zanahoria. Las hortalizas con la misma frecuencia de compra, pero con cantidades promedio de 0,5 kilos son: Ají picante, ajo, apio, cebollín, cilantro, col, esparrago, espinaca y perejil.

Una de las formas de establecer la importancia de las hortalizas es considerar la proporción del presupuesto familiar que destinan a la compra de estas. Con el análisis de frecuencias, se encontró que las hortalizas ocupan un lugar intermedio en la asignación de presupuesto de compras de las familias debido a que los consumidores en promedio destinan para su consumo el 31 y el $40 \%$ de su presupuesto.

En cuanto a la dinámica que ha tenido el consumo de hortalizas frescas en los hogares de la Región Caribe en los últimos cinco años el 54,5\% de los encuestados manifestaron no haber tenido cambios en las cantidades consumidas, sin embargo, el 38,3\% afirman que disminuyó y el 7,1\% aumentó su consumo.

Las razones de la disminución del consumo se deben principalmente a los precios altos en un $51,4 \%$ de los que respondieron la pregunta, seguido de que no la prefieren los niños en $31,4 \%$ y es sustituidos por otras opciones de alimentos en un $11,4 \%$ y solo lo consumen los adultos en $2,9 \%$ en tanto que el $2,9 \%$ solo consumen lo que ellos consideran necesario.

Los factores que motivan el aumento del consumo de acuerdo a la encuesta, el valor nutritivo de estas y beneficios que aporta a la salud, representan el mayor porcentaje con un valor de $89 \%$, mientras que el $10,3 \%$ se lo atribuyen a los precios favorables que permiten su compra y el $0,7 \%$ al aumento de los ingresos, estos resultados evidencian la presencia de la ley de Engel que argumenta que ante aumentos en una unidad monetaria en el ingreso del consumidor destina un máximo del $7 \%$.

La percepción acerca de la importancia que le dan las personas al consumo de hortalizas teniendo en cuenta la edad del consumidor se presenta en la tabla 1, esta información permite apreciar el concepto que tiene el consumidor acerca del grado de importancia que le dan las familias al consumo de hortalizas. Se aprecia que en general todos los rangos de edades encuestados le confieren un grado alto de importancia al consumo de hortalizas sin embargo las edades mayores de 50 años son los que más importancia le dan al consumo de estas. Esto permite inferir que en estas circunstancias se debería incentivar en la población infantil y los jóvenes para que aumenten el consumo y así poder aumentar la oferta, si se quiere que este renglón de la producción sea sostenible.

Para caracterizar al consumidor de hortalizas se usó como variable el sitio de compra, que a su vez se relacionó con el estrato social y nivel de escolaridad. 
Tabla 1. Importancia del consumo de las hortalizas de acuerdo con la edad del consumidor en la Región Caribe.

\begin{tabular}{cccccc}
\hline Edad & \multicolumn{4}{c}{ Importancia del Cosumo } & Total \\
& Poca & Baja & Mediana & Alta & \\
\hline $20-30$ & 4 & 7 & 29 & 47 & 87 \\
$31-40$ & 3 & 7 & 15 & 31 & 56 \\
$41-50$ & 3 & 11 & 26 & 45 & 85 \\
$\begin{array}{c}\text { Mayor } \\
\text { de 50 }\end{array}$ & 5 & 5 & 14 & 56 & 80 \\
\hline Total & $\mathbf{1 5}$ & $\mathbf{3 0}$ & $\mathbf{8 4}$ & $\mathbf{1 7 9}$ & $\mathbf{3 0 8}$ \\
\hline
\end{tabular}

Fuente: Elaboración propia, Agrosavia 2018.

En la tabla 2, se presenta la relación de esta variable con el nivel de ingreso, resultando que, aunque existen diversas opciones de sitio compra de hortalizas frescas, el consumidor es quien lo elige de acuerdo con el nivel de ingresos. En tal sentido, todos los encuestados visitan los supermercados para realizar compras en algún momento, sin embargo, los que disponen de más ingresos son los que prefieren siempre el supermercado como sitio de compra para las hortalizas. Los consumidores con niveles de ingreso bajo compran en el mercado público y en pequeñas proporciones en la tienda de la esquina.

Tabla 2. Sitio de compra de hortalizas por nivel de ingresos Región Caribe

\begin{tabular}{|c|c|c|c|c|c|}
\hline \multicolumn{6}{|c|}{ Rango de ingresos en miles de pesos COP } \\
\hline $\begin{array}{l}\text { Lugar de } \\
\text { compra }\end{array}$ & $\begin{array}{c}\text { Menor } \\
500\end{array}$ & $\begin{array}{l}500- \\
1000\end{array}$ & $\begin{array}{l}1000- \\
5000\end{array}$ & $\begin{array}{c}\text { Mayor } \\
5000\end{array}$ & Total \\
\hline $\begin{array}{l}\text { Supermer- } \\
\text { cado }\end{array}$ & 13 & 51 & 64 & 14 & 142 \\
\hline $\begin{array}{l}\text { Tienda de } \\
\text { la esquina }\end{array}$ & 4 & 18 & 14 & 0 & 36 \\
\hline $\begin{array}{l}\text { Carretillero } \\
\text { de barrio }\end{array}$ & 6 & 12 & 4 & 0 & 22 \\
\hline $\begin{array}{l}\text { Mercado } \\
\text { publico }\end{array}$ & 30 & 50 & 25 & 2 & 107 \\
\hline $\begin{array}{l}\text { Productor } \\
\text { directo }\end{array}$ & 0 & 1 & 0 & 0 & 1 \\
\hline Total & 53 & 132 & 107 & 16 & 308 \\
\hline
\end{tabular}

Fuente: Elaboración propia, Agrosavia 2018.
Con relación a la incidencia del grado de escolaridad en las decisiones del sitio de compra, se encontró que el $64,2 \%$ de los consumidores con grado universitario prefieren el supermercado, seguido de mercado público con $21,16 \%$; y tienda de la esquina con un $13,13 \%$ mientras que un pequeño porcentaje $(1,45 \%)$ eligen comprar las hortalizas en el carretillero de barrio o productor directo. Los consumidores sin título universitario prefieren realizar la compra de las hortalizas en el mercado público. Otra variable para entender las decisiones del consumo fue considerar la edad de los consumidores con el sitio de compra, en tal sentido, quienes presentan mayor edad prefieren al mercado público y a los supermercados (Tabla 3)

Tabla 3. Sitio de compra de hortalizas por rango de edad en la Región Caribe

\begin{tabular}{|c|c|c|c|c|c|}
\hline \multicolumn{6}{|c|}{ Rango de edad en años } \\
\hline $\begin{array}{l}\text { Lugar de } \\
\text { compra }\end{array}$ & 20-30 & $31-40$ & $41-50$ & $\begin{array}{l}\text { Mayor } \\
\text { de } 50\end{array}$ & Total \\
\hline $\begin{array}{l}\text { Supermer- } \\
\text { cado }\end{array}$ & 47 & 26 & 34 & 35 & 142 \\
\hline $\begin{array}{l}\text { Tienda de la } \\
\text { esquina }\end{array}$ & 11 & 9 & 11 & 5 & 36 \\
\hline $\begin{array}{l}\text { Carretillero } \\
\text { de barrio }\end{array}$ & 9 & 4 & 9 & 0 & 22 \\
\hline $\begin{array}{l}\text { Mercado } \\
\text { publico }\end{array}$ & 19 & 17 & 31 & 40 & 107 \\
\hline $\begin{array}{l}\text { Productor } \\
\text { directo }\end{array}$ & 1 & 0 & 0 & 0 & 1 \\
\hline Total & 87 & 56 & 85 & 80 & 308 \\
\hline \multicolumn{6}{|c|}{ Fuente: Elaboración propia, Agrosavia 2018.} \\
\hline \multicolumn{6}{|c|}{$\begin{array}{l}\text { Es importante saber cuáles son las razones } \\
\text { que Ilevan al consumidor a elegir el sitio de } \\
\text { compra las cuales se pueden apreciar en } \\
\text { tabla } 4 \text {. En primer lugar, los que prefieren los } \\
\text { supermercados lo hacen por la calidad que } \\
\text { corresponde a un } 25 \% \text { de la muestra } \\
\text { encuestada, los que compran en el mercado } \\
\text { público lo hacen porque los precios son } \\
\text { favorables que corresponden al } 20 \% \text { de la } \\
\text { muestra encuestada. Los demás prefieren otros } \\
\text { sitios como la tienda de la esquina por cercanía } \\
\text { a la casa. En el caso del carretillero del barrio }\end{array}$} \\
\hline
\end{tabular}


que es muy típico en la Región Caribe lo hace porque encuentran todas las hortalizas que necesitan de forma oportuna. Otro enfoque para analizar las preferencias del consumidor por el sitio de compra es tener en cuenta los cambios del sitio por la influencia del género, lo cual puede apreciarse en la figura 1. Esta misma variable en función del género muestra que tanto hombres y mujeres tiene como primera opción de compra los supermercados y el mercado público (Figura 1), al observarlo de manera desagregada se encontró que, en mayor proporción, las mujeres $(22,7 \%)$ compran en estos sitios y los hombres lo hacen en una proporción más baja (11\%). Igualmente, en el caso de la compra en los supermercados la mujer lo hace en una proporción mayor $(33,7 \%)$, en tanto que el hombre lo hace en una proporción más baja (12\%).

Tabla 4. Razones de preferencia del sitio de compra de hortalizas en los principales mercados de la Región Caribe.

\begin{tabular}{|c|c|c|c|c|c|c|}
\hline $\begin{array}{l}\text { Lugar de } \\
\text { compra }\end{array}$ & Calidad & $\begin{array}{c}\text { Encuentra } \\
\text { todas }\end{array}$ & $\begin{array}{c}\text { Precio } \\
\text { favorable }\end{array}$ & $\begin{array}{l}\text { Cercanía } \\
\text { a la casa }\end{array}$ & $\begin{array}{c}\text { Mejor } \\
\text { presentación y } \\
\text { medida }\end{array}$ & Total \\
\hline Supermercado & 78 & 30 & 19 & 10 & 5 & 142 \\
\hline Tienda & 0 & 10 & 11 & 15 & 0 & 36 \\
\hline Carretillero & 2 & 12 & 7 & 1 & 0 & 22 \\
\hline $\begin{array}{l}\text { Mercado } \\
\text { publico }\end{array}$ & 7 & 29 & 63 & 8 & 0 & 107 \\
\hline Productor & 0 & 0 & 1 & 0 & 0 & 1 \\
\hline Total & 87 & 81 & 101 & 34 & 5 & 308 \\
\hline
\end{tabular}

Fuente: Elaboración propia, Agrosavia 2018.

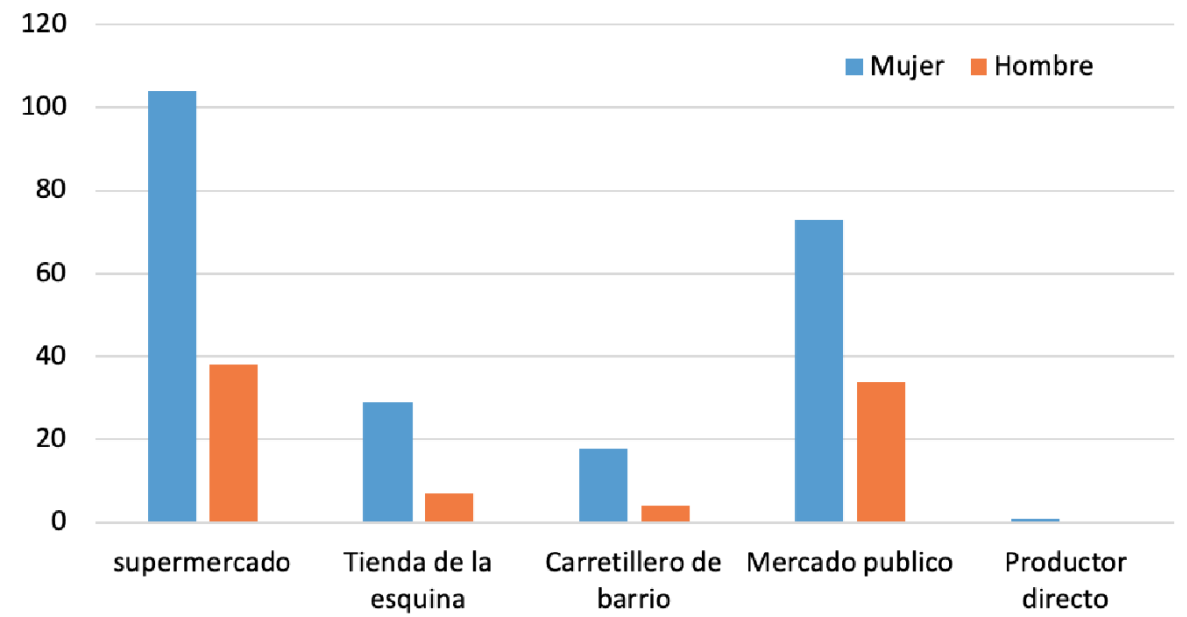

Figura 1. Sitio de compra de hortalizas frescas de acuerdo al género en el Caribe Colombiano.

En el caso de la tienda de la esquina, la mujer participa con un 9\%, mientras que el hombre lo hace en una proporción muy baja $(2,2)$. Lo anterior permite pensar en establecer nuevas estrategias de comercialización para el abastecimiento de estos puntos, especialmente las buenas prácticas de conservación de los productos y mejoras en la presentación de estos, a fin de que sea más amigable la compra para las mujeres que lo hacen en una proporción mayor. Estos resultados son similares a los obtenidos por Vera et al. (2011) en donde reportan que los estratos altos prefieren los supermercados y ferias como 
lugar de compras de hortalizas, en tanto que los de menores ingresos compran en las tiendas cerca a sus casas; con relación al género en este estudio las mujeres consumen más hortalizas que los hombres.

Otro aspecto analizado dentro del estudio fueron las razones o motivos que influyen en la disminución del consumo de hortalizas, los más sobresaliente fueron los precios altos, seguido el bajo consumo de estas en los niños y los jóvenes y un alto porcentaje que no respondió la pregunta. Esta situación reitera que la población adulta es la que más prefiere las hortalizas dentro su dieta.

Los factores o determinantes del aumento del consumo de hortalizas en la Región Caribe están asociados a las variables valor nutritivo, beneficios a la salud y los precios favorables y muy poco se ve influenciado por el aumento de los ingresos, lo que significa que las proporciones del gasto destinado al consumo de hortalizas se mantiene constante, por lo tanto, no es posible aumentar el consumo por la vía de los ingresos. Sin embargo, lo que se puede evidenciar, es que el consumo en una alta proporción ha permanecido igual (50,1\%), esto significa que existen pocas posibilidades de aumentar el consumo de hortalizas frescas; tal como está la situación actualmente, con campañas que estimulen el hábito del consumo es posible aumentar las tasas de crecimiento.

Con relación a la frecuencia del consumo de hortalizas en la Región Caribe, se puede apreciar que los consumidores prefieren hacer las compras de hortalizas cada siete días lo que corresponde al $51 \%$ de la muestra encuestada, seguido de cada 15 días que participa con el $24 \%$, diario y cada 30 días con el 13,6 y $10,1 \%$ respectivamente. Tal vez se explique esta frecuencia dada la naturaleza de los productos de carácter perecedero, lo que implica que la vida de anaquel no es muy larga y, por tanto, los consumidores prefieren intervalos más cortos. Con relación a las posibilidades de aumentar las cantidades consumidas, el
$49 \%$ de los encuestados afirma no querer aumentar el consumo y las razones que expone son: Bajo consumo por parte de los jóvenes, un porcentaje muy bajo (4\%) afirman que por los precios no pueden aumentar el consumo; otrarazónesquesoloconsumenlasque consideran necesario y forman parte de la dieta como las cebollas, cilantro, zanahoria y tomate. Las demás no las consideran importantes. El 51\% de los encuestados afirma tener disposición para aumentar el consumo de las hortalizas y lo harían por su calidad o valor nutritivo, beneficios para la salud y los precios favorables. La anterior afirmación se puede validar si se relaciona el aumento del consumo de las hortalizas con el nivel de ingresos. En este caso para los niveles de ingreso entre $\$ 1.000 .000$ y $\$ 5.000 .000$ COP en un $20 \%$ estarían dispuestos a aumentar el consumo, seguido del rango de ingresos entre $\$ 500.000$ y $\$ 1.000 .000$ COP que corresponden al $6,8 \%$ de la muestra encuestada; pero se aprecia que los niveles de ingreso mayores a $\$ 5.000 .0000$ COP estarían dispuestos a aumentar el consumo, pero en una proporción de solo el 2,5\% de la muestra encuestada.

Al relacionar el aumento del consumo de las hortalizas con los ingresos de las familias, los datos de las encuestas arrojaron que indistintamente del nivel de ingreso los encuestados están dispuesta a aumentar el consumo de las hortalizas en las siguientes proporciones: ingresos menores a $\$ 500.000$ un $13 \%$; el rango de ingreso entre $\$ 500.000$ COP y $\$ 1.000 .000$ COP corresponde al $42 \%$, los niveles de ingreso entre $\$ 1.000 .000$ COP y $\$ 5.000 .000$ COP en un $39 \%$ y el de más $\$ 5.000 .000$ COP en un 5\%, lo que significa que niveles de ingreso intermedio serían los que más estarían dispuestos a aumentar el consumo.

Una de las variables que se analizaron fue la disposición a pagar un precio mayor por hortalizas de mejor calidad obtenidas bajo un proceso de buenas prácticas agrícolas. Las respuestas muestran que $30 \%$ no estaría dispuesto a pagar un precio adicional, en tanto que el $70 \%$ si lo hicieran. 
Igualmente, las familias con rango de ingresos $\$ 500.000$ COP y $\$ 1.000 .000$ COP son las que respondieron estar dispuestas a pagar más por hortalizas de buena calidad, que corresponde al $41 \%$ de la muestra encuestada. Del grupo de familias con ingresos superiores a $\$ 5.000 .000$ COP solo el $7 \%$ estarían dispuestas a pagar un precio más alto por hortalizas de buena calidad. Las razones para no incurrir en este pago adicional son: en primer lugar, por motivos económicos relacionados con los bajos ingresos de la familia, con un $22,1 \%$ de la muestra encuestada; la segunda razón, es porque no cree que las buenas prácticas agrícolas mejoren la calidad, sino que se constituye en una excusa para aumentar los precios, esta afirmación la hizo el 8,4\% de la muestra encuestada; y en tercer lugar, un $2,3 \%$ cree que este costo lo debe asumir el gobierno. Algunos encuestados manifiestan que es obligación del productor vender alimentos sanos y de buena calidad $y$, por este motivo, no tienen por qué transferir este costo al consumidor, un $67 \%$ no respondió esta pregunta.

Al comparar los resultados de este estudio con los obtenidos en Valdivia Chile (Vera et al., 2011), para las hortalizas se puede apreciar que se llega a conclusiones muy similares. En principio, el estudio realizado para la Región Caribe se encontró que las hortalizas que más prefieren los consumidores son zanahorias, la cebolla blanca, la ahuyama y el tomate en promedio 1 kilo por compra. Por su parte, en el estudio de Chile las hortalizas que mayor consumen son tomates y zanahorias, con una preferencia del consumo del $82 \%$. Referente a la frecuencia de compra, tanto en el caso del estudio de chile como en el de la Región Caribe es semanal, esto se explica por la naturaleza de las hortalizas por el carácter perecedero que hace que las compras no sean tan distantes. Con relación al género, en el caso de Chile el 55\% de los consumidores correspondieron a mujeres y un $45 \%$, a varones, mientras que la región Caribe el $69 \%$ de los consumidores son mujeres y el $31 \%$ son hombres. En los dos casos el mayor porcentaje de consumidores lo ocupan las mujeres. En la única variable que hubo diferencias en los dos estudios fue la edad de los consumidores; en el caso de chile el rango que más se repite es entre 30 y 40 años, en tanto la Región Caribe la edad que más se repite es entre 40 y más de 50 años. En La región Caribe los consumidores son de mayor edad y los bajos consumos están en los niños y jóvenes.

En general se observa que el consumo de hortalizas frescas en la Región Caribe es bajo Con relación al total nacional el consumo 45,75 gramos/persona/ día en tanto que para la Región Caribe da un promedio de 3,69 gramos por persona/día, muy por debajo al consumo de Alemania de 255 g/día por persona. El análisis de varianza se hizo para realizar las comparaciones de la variable cuantitativa, en este caso, cantidades demandadas de hortalizas a través de las variables categóricas: genero (hombre o mujer), tamaño de la familia y nivel de ingreso lo cual se presenta en la tabla 5.

Tabla 5. ANOVA de un factor: respuesta del consumo promedio de hortalizas ante el género del consumidor, la composición familiar y el nivel de ingresos.

\begin{tabular}{|c|c|c|c|}
\hline & Variable & $\begin{array}{c}\text { Consumo } \\
\text { promedio } \\
\text { kg }\end{array}$ & $\begin{array}{l}\text { Porcentaje de } \\
\text { Significancia }\end{array}$ \\
\hline \multirow{2}{*}{ Genero } & Mujer & 3,4 & \multirow{2}{*}{0,18} \\
\hline & Hombre & 1,2 & \\
\hline \multirow{7}{*}{$\begin{array}{l}\text { Tamaño } \\
\text { de familia }\end{array}$} & 1 & 0,34 & \multirow{7}{*}{0,17} \\
\hline & 2 & 1,5 & \\
\hline & 3 & 4 & \\
\hline & 4 & 5 & \\
\hline & 5 & 1,3 & \\
\hline & $\begin{array}{c}\text { Mayor de } \\
6\end{array}$ & 10 & \\
\hline & Total & 3,69 & \\
\hline \multirow{4}{*}{$\begin{array}{c}\text { Nivel } \\
\text { Ingreso } \\
\text { en miles } \\
\text { de pesos } \\
\text { COP }\end{array}$} & $\begin{array}{c}\text { Menor de } \\
500\end{array}$ & 1,35 & \multirow{4}{*}{0,14} \\
\hline & $\begin{array}{c}500 \\
-1000\end{array}$ & 4,37 & \\
\hline & $\begin{array}{c}1000 \\
-5000\end{array}$ & 3,05 & \\
\hline & $\begin{array}{c}\text { Mayor de } \\
5000\end{array}$ & 0,72 & \\
\hline
\end{tabular}

Fuente: Elaboración propia, Agrosavia 2018. 
La Región Caribe presenta un mayor consumo de hortalizas en las mujeres en comparación con los hombres, se estima que en promedio una mujer consume a la semana 3,4 kilos, en tanto que los hombres consumen 1,2 kilos a la semana.

Para el caso del consumo familiar la encuesta presentó un promedio de número de miembros de la familia de 1 hasta más de 4 pero el grupo que mayor consumo de hortalizas tuvo fue la familia compuesta por más de seis miembros, peroes interesantequeel promediode miembros por familia de cuatro consume 5 kilos por semana equivalente a 1,25 kilos por persona.

Por su parte, al aplicar la misma variable, pero ahora en función de los ingresos se observa que los hogares con ingresos intermedios cuentan con el consumo más elevado de hortalizas frescas (rango de ingresos entre $\$ 500.000$ - \$1.000.000 COP) correspondiente a 4,37 kilos por semana.

Conclusiones similares a las de Vera et al. (2011) y las del presente trabajo, pero con otras especies se encontraron en el trabajo realizado por Montaño et al. (2015) sobre caracterización del consumidor de calamar en Baja California Sur, donde el $63 \%$ de los consumidores son mujeres, y la edad promedio se encuentra en un rango de 21 a 39 años con una participación del $54 \%$ de la muestra encuestada. En el caso de la Región Caribe la edad promedio se encuentra en el rango de 40 a 50 años, la moda es de 42 años. El nivel de ingresos promedio mensual de las familias está en un rango de 500.000 y $1,000.000$ de pesos colombianos, que corresponde al $43 \%$ de la muestra encuestada.

\section{CONCLUSIONES}

El consumidor de la Región Caribe revela preferencias sobre las decisiones para el consumo de las hortalizas que desea comprar las culés se fundamentan en atributos como frescura, valor nutricional, beneficios a la salud y bajos precios, en este sentido las que más prefieren son la zanahoria, tomate, cebolla blanca, ahuyama, cebolla roja, lechuga y remolacha. La edad de los consumidores influye mucho al momento de decidir por la compra de hortalizas, siendo las edades de los 40 años en adelante los que compran hortalizas en mayores cantidades y frecuencia.

El sitio de venta preferido por los consumidores de hortalizas es el mercado público, dado a que los precios son más favorables y encuentran todas las verduras a comprar. Existe una relación entre la decisión del sitio de compra y el nivel de ingreso, los niveles más altos prefieren los supermercados y los estratos más bajos los mercados públicos o plazas de mercado.

Las familias con niveles de ingresos intermedio es decir los que tiene entre $\$ 500.000$ COP y $\$ 1.000 .000$ COP mensuales son los que presentan mayor proporción para aumentar el consumo, sin embargo, se recomienda continuar con estudio que indaguen acerca de los determinantes del aumento del consumo y la disposición por parte del consumidor.

\section{Conflicto de Intereses}

Los autores declaran que es un trabajo orginal y no existío conflicto de intereses de ningún tipo en la elaboración y publicación del manuscrito.

\section{AGRADECIMIENTOS}

Al Ministerio de Agricultura y Desarrollo Rural de Colombia por la financiación del proyecto que dio origen a esta información a través de la agenda de investigación de Agrosavia. A los investigadores de Agrosavia del Centro de Investigacion Turipaná por el apoyo en las encuestas.

\section{REFERENCIAS}

Case, K., Fair, R. y Oster, S. 2012. Principios de Microeconomía. México: Editorial Pearson Educación. 
FAO 2002. Alimentos inocuos y nutritivos para los consumidores. Cumbre Mundial sobre alimentación.

Hair, J., Anderson, R., Tatham, R. y Black, W. 1999. Análisis multivariante. 5.a ed. Madrid: Pearson Prentice Hall.

Martín, V. 2005. Hábitos de compra y consumo de frutas y hortalizas. En revista Distribución y consumo. Universidad Complutense de Madrid

Miller, N. 2006. "Notes on Microeconomic Theory". Harvard University. USA. http://www.business.illinois.edu/nmiller/ documents/notes/firsthalf.pdf

Malhotra, N. 1997. Investigación de mercados: un enfoque práctico. 2.a ed. México: Prentice-Hall Hispanoamericana.

Ministerio de Salud y Protección Social, 2013. Perfil Nacional de consumo de Frutas y verduras.

https://www.minsalud.gov.co/sites/rid/ Lists/BibliotecaDigital/RIDE/VS/PP/ $\underline{\text { SNA/perfil-nacional-consumo-frutas-y- }}$ verduras-colombia-2013.pdf

Montaño, M., Hernandez, G., Mayoral, J., Mesías, D. y Ávila, A. 2015. Caracterización del consumidor de calamar gigante en Baja California Sur, México. Revista Corpoica Ciencia. Tecnología. Agropecuaria. 16(1).

Nicholson, W. 1997. Teoría Microeconómica "Principios y Aplicaciones" Editorial McGraw Hill.
Parkin, M. y Loria, E. 2006. Microeconomía versión Para Latinoamérica. México: Pearson Educación.

Reynolds, L. 2013. Percepciones y preferencias del consumidor de palmito fresco. Caso: unión de asociaciones de productores de plantines y palmito. Revista Perspectivas, (32).

http://www.scielo.org.bo/ scielo.php?pid=S1994$\underline{37332013000200003 \& \text { script }=\text { sci }}$ arttext\&tlng=en

Rodríguez, J. y Mora, R. 2001. Estadística informática: casos y ejemplos con el SPSS. 1. a ed. Alicante: Universidad de Alicante.

Samuelson, P. 1948. Consumption theory in terms of revealed preferences. Económica 15: 243- 251.

Salazar, J. y Vásquez, F. 2010. El Legado De Samuelson La preferencia revelada. Revista Internacional de Sociología (RIS), 68(3), 797-804 https://doi.org/10.3989/ris.2010.03.24

Torres $\mathbf{M}, 2$ 2016. Tamaño de una muestra para una investigación de mercado. En Boletín Electrónico No. 02. Facultad de Ingeniería - Universidad Rafael Landívar, Guatemala.

Vera, M., Fajardo. G. y Echavarria, R, 2011. Actitudes y preferencias del consumidor de frutas y hortalizas en estado fresco. Ciudad de Valdivia. Región de los ríos. Agro Sur Vol. 39(1) 35-45 